\title{
Guest Editorial: Special Issue on Fetal Hemodynamics
}

\section{Developmental Fetal Cardiovascular Biomechanics in the 21st Century: Another Tipping Point}

The relationships between structure and function during cardiovascular morphogenesis have fascinated biologists, anatomists, and clinicians for centuries due to the observable and carefully orchestrated transformation of simple cellular structures into highly ordered and dynamically functioned organs and the broad spectrum of malformations that occur and produce congenital cardiovascular defects. For example, in 1932 Harvard anatomist Bremer described two spiral flow streams within the primitive, unseptated heart based on observations of moving blood cells. ${ }^{3}$ For many years, it was thought that the paths of these streams predetermined the formation of the spiral aortopulmonary septum, the asymmetric mature aortic arch and pulmonary arteries, and a subset of congenital cardiac defects. Contemporary studies using fluorescent dye injections to map intracardiac flow streams have shown that this relationship is much more complex $^{26}$ and involves the interactions between migrating, extracardiac neural crest cells, ${ }^{13}$ cell-matrix, ${ }^{22}$ and paracrine regulation. ${ }^{7}$

The importance of biomechanical loading forces in the morphogenesis of the great-vessels, valves and ventricles and in the origins of cardiac malformations is now well established though the fundamental biological mechanisms regulating these transduction and remodeling processes are largely undefined. ${ }^{21,23}$ Unlike the mature adult cardiovascular system, cardiovascular anatomy and tissue-level cellular and extracellular structure is highly dynamic featuring significant remodeling in response to altered external mechanical loading. ${ }^{11,24}$ Decades of research have shown that disrupting the normal flow patterns solely by mechanical intervention generates a spectrum of cardiovascular defects. ${ }^{6,8,12}$

The clinical relevance of defining the origins of congenital cardiovascular malformations relates to the high incidence of heart defects in humans (approximately $1 \%$ of live births and a much higher percentage of the pregnancies that end unsuccessfully in the first trimester), the requirement for medical and surgical intervention in at least $25 \%$ of these children in the first year of life, and the life-long impact of congenital heart disease on health, employment, and subsequent generations. ${ }^{16,19}$ The broad based and international investigation that integrates comparative and developmental biology, the molecular genetics of inverte- brate and vertebrate animal models, multimodal and predictive bioengineering approaches early ${ }^{10}$ and expanding data sets from advanced human fetal imaging $^{20}$ and intervention ${ }^{18}$ has reached the tipping point where observed congenital cardiovascular malformations can now be coupled to unique sequences of spatio-temporal events that trigger predictable altered developmental trajectories. One of the outcomes of this expanding knowledge base will be altered clinical management strategies designed to redirect developing structures towards preferred states that optimize fetal cardiovascular structure and function, ${ }^{18}$ postnatal successful interventions, and improved quality of life.

The present Special Issue highlights additional and multi-disciplinary themes relevant to the dynamic relationships between comparative developmental cardiovascular biomechanics, morphogenesis, and malformations. Balasubramanian and $\mathrm{Tacy}^{2}$ present an up-to-date perspective on the truly fascinating capabilities of ultrasound in functional cardiac imaging, including the quantification of intramyocardial strains and perfusion and acquisition of four-dimensional anatomical data sets. Professor Burggren ${ }^{5}$ explores the role of early embryonic heart in driving angiogenesis using comparative and integrative biologic approaches. His data suggest that the one function of the early pulsatile embryonic heart is to stimulate angiogenesis by creating cyclic shear/strain on the endothelial linings of sprouting vessels rather than the transport of respiratory gases and nutrients. ${ }^{4}$ Johnson et al. ${ }^{14}$ describe the maturation of quantitative cardiac function in the univentricular embryonic zebrafish using in vivo particle image velocimetry measurements at multiple developmental time-points. Leinana et al. ${ }^{15}$ provide realistic computational fluid dynamics simulations of the pulsatile flow at the bifurcation of the umbilical vein and ductus venosus bifurcation where critical changes in the distribution of blood flow can impact cardiac and fetal outcomes. Giancarlo et al. ${ }^{9}$ investigate umbilical arterial biomechanics that are essential for accurate system-level modeling of fetal-placental circulatory interactions. Finally, Luria et al. ${ }^{17}$ analyzed fetal growth restriction by integrating experimental Doppler velocity waveforms with lumped parameter mathematical modeling of the fetal circulation for the assessment of fetal 
hemodynamics in a broad spectrum of gestational ages.

Hopefully these papers provide additional insights into the expanding frontiers of embryonic and fetal developmental cardiovascular hemodynamics both relevant and essential to our understanding of the causes and consequences of congenital cardiovascular defects. We are now in the rapidly advancing era of human fetal diagnosis and therapies for a range of congenital malformations ${ }^{1}$ and clinicians will use the insights gained from these bioengineering-led studies to base both their timing and therapeutic approach in order to modify or reverse altered development trajectories. Continued advances in high-resolution functional cardiac magnetic resonance imaging ${ }^{25}$ will further support these strategies. The rapid expansion of these complex and multi-modal data sets will require a parallel expansion and optimization of computational predictive technologies that can integrate these data in order to guide fetal and post-natal interventions towards optimal long-term outcomes.

\section{CONFLICT OF INTEREST}

Authors have no disclosure of interest.

\section{REFERENCES}

${ }^{1}$ Arzt, W., and G. Tulzer. Fetal surgery for cardiac lesions. Prenat. Diagn. 31:695-698, 2011.

${ }^{2}$ Balasubramanian, S., and T. A. Tacy. Functional imaging in fetal echocardiography. Cardiovasc. Eng. Technol. 2013. doi:10.1007/s13239-013-0132-z.

${ }^{3}$ Bremer, J. L. The presence and influence of two spiral streams in the heart of the chick embryo. Am. J. Anat. 49:409-440, 1932.

${ }^{4}$ Burggren, W. W. What is the purpose of the embryonic heart beat? Or how facts can ultimately prevail over physiological dogma. Physiol. Biochem. Zool. 77:333-345, 2004.

${ }^{5}$ Burggren, W. W. Cardiovascular development and angiogenesis in the early vertebrate embryo. Cardiovasc. Eng. Technol. 2013. doi:10.1007/s13239-013-0118-x.

${ }^{6}$ Clark, E. B., and G. C. Rosenquist. Spectrum of cardiovascular anomalies following cardiac loop constriction in the chick embryo. Birth Defects Orig. Artic. Ser. 14:431442, 1978.

${ }^{7}$ de la Pompa, J. L., and J. A. Epstein. Coordinating tissue interactions: notch signaling in cardiac development and disease. Dev. Cell. 22:244-254, 2012.

${ }^{8}$ Gessner, I. H. Spectrum of congenital cardiac anomalies produced in chick embryos by mechanical interference with cardiogenesis. Circ. Res. 18:625-633, 1966.

${ }^{9}$ Giancarlo, P., K. Laganà, F. Gervaso, S. Rigano, and E. Ferrazzi. How do cord compressions affect the umbilical venous flow resistance? An in vitro investigation of the biomechanical mechanisms. Cardiovasc. Eng. Technol. 2013. doi:10.1007/s13239-013-0131-0.

${ }^{10}$ Goenezen, S., M. Y. Rennie, and S. Rugonyi. Biomechanics of early cardiac development. Biomech. Model. Mechanobiol. 11:1187-1204, 2012.

${ }^{11}$ Hogers, B., and M. C. DeRuiter, A. C. Gittenberger-de Groot, and R. E. Poelmann. Extraembryonic venous obstructions lead to cardiovascular malformations and can be embryo lethal. Cardiovasc. Res. 41:87-99, 1999.

${ }^{12} \mathrm{Hu}$, N., D. A. Christensen, A. K. Agrawal, C. Beaumont, E. B. Clark, and J. A. Hawkins. Dependence of aortic arch morphogenesis on intracardiac blood flow in the left atrial ligated chick embryo. Anat. Rec. (Hoboken) 292:652-660, 2009.

${ }^{13}$ Hutson, M. R., and M. L. Kirby. Neural crest and cardiovascular development: a 20-year perspective. Birth Defects Res. C Embryo Today 69:2-13, 2003.

${ }^{14}$ Johnson, B. M., M. G. Deborah, and L. P. Dasi. The transitional cardiac pumping mechanics in the embryonic heart. Cardiovasc. Eng. Technol. 2013. doi:10.1007/s13239013-0120-3.

${ }^{15}$ Leinana, P. R., T. Kiserudb, and L. R. Hellevika. Human ductus venosus velocity profiles in the first trimester. Cardiovasc. Eng. Technol. 2013. doi:10.1007/s13239-013-0133-y.

${ }^{16}$ Loffredo, C. A. Epidemiology of cardiovascular malformations: prevalence and risk factors. Am. J. Med. Genet. 97:319-325, 2000.

${ }^{17}$ Luria, O., J. Bar, J. Shalev, M. Kovo, A. Golan, and O. Barnea. Inverse solution of the fetal-circulation model based on ultrasound Doppler measurements. Cardiovasc. Eng. Technol. 2013. doi:10.1007/s13239-013-0153-7.

${ }^{18}$ McElhinney, D. B., W. Tworetzky, and J. E. Lock. Current status of fetal cardiac intervention. Circulation 121:12561263, 2010.

${ }^{19}$ Moller, J. Prevalence and incidence of cardiac malformation. In: Perspectives in Pediatric Cardiology: Surgery of Congenital Heart Disease: Pediatric Cardiac Care Consortium, 1984-1995, edited by J. Moller. Armonk, NY: Futura Publishing, 1998, pp. 19-26.

${ }^{20}$ Phoon, C. K. Circulatory physiology in the developing embryo. Curr. Opin. Pediatr. 13:456-464, 2001.

${ }^{21}$ Poelmann, R. E., A. C. Gittenberger-de Groot, and B. P. Hierck. The development of the heart and microcirculation: role of shear stress. Med. Biol. Eng. Comput. 46:479-484, 2008.

${ }^{22}$ Sizarov, A., W. H. Lamers, T. J. Mohun, N. A. Brown, R. H. Anderson, and A. F. Moorman. Three-dimensional and molecular analysis of the arterial pole of the developing human heart. J. Anat. 220:336-349, 2012.

${ }^{23}$ Taber, L. A., B. B. Keller, and E. B. Clark. Cardiac mechanics in the stage-16 chick embryo. J. Biomech. Eng. 114:427-434, 1992.

${ }^{24}$ Tobita, K., J. B. Garrison, L. J. Liu, J. P. Tinney, and B. B. Keller. Three-dimensional myofiber architecture of the embryonic left ventricle during normal development and altered mechanical loads. Anat. Rec. A Discov. Mol. Cell Evol. Biol. 283:193-201, 2005.

${ }^{25}$ Votino, C., J. Jani, N. Damry, H. Dessy, X. Kang, T. Cos, et al. Magnetic resonance imaging in the normal fetal heart and in congenital heart disease. Ultrasound Obstet. Gynecol. 39:322-329, 2012.

${ }^{26}$ Yoshida, H., F. Manasek, and R. A. Arcilla. Intracardiac flow patterns in early embryonic life. A reexamination. Circ. Res. 53:363-371, 1983. 
Kerem Pekkan

Pediatric Cardiovascular Fluid Mechanics Laboratory, Carnegie Mellon University, 700 Technology Drive, Pittsburgh,

PA 15219, USA

Electronic mail: kpekkan@andrew.cmu.edu

Mechanical Engineering Department, Koç University, Rumeli feneri Yolu, Istanbul 34450, Turkey

Electronic mail: kpekkan@ku.edu.tr
Bradley B. Keller

Department of Pediatrics, University of Louisville, 302 East Muhammad Ali Blvd, Louisville, KY 40202, USA

Electronic mail: Brad.Keller@louisville.edu 